\title{
Comparative Study of Conservative Vs Surgical Management of Clavicle Fractures
}

\author{
Vivek Phanswal \\ Assistant Professor, Department of Orthopedics, Noida International Institute of Medical Sciences, \\ Gautam Buddha Nagar, Uttar Pradesh
}

\begin{abstract}
Background: Clavicle Fractures is one of the common fractures of upper limb accounting for approximately $40 \%$ of all shoulder fractures. Till recently all clavicle fracture were treated conservatively, but now interest in surgical management is rising. This study was carried out to see if surgical management outweighs the conservative treatment of Fractures Of Clavicle.

Methods: A total of 40 cases satisfying inclusion and exclusion criteria were included in the study. Alternate patients were allocated to operative and conservation groups. In total 20 patients were operated operatively and 20 patients were treated conservatively. Outcome was analysed in terms of radiological union and functional outcome of the patient. Constant and Murley score was used as a score to evaluate final outcome.
\end{abstract}

Results: In this study, the 20 patients who were operated upon had an average union time of 7.8 weeks; 1 patient had delayed fracture union by 12 wks. 20 patients in conservative group had normal union of fractures with 1 patient going into non-union. Average union time in the conservative group was 9.4 weeks, which was more than the 7.8 weeks seen in the group treated operatively. Mal-union was present in 7 of the 20 patients treated conservatively, and 5 of these 7 had a visible deformity. Out of these patients with mal-union 1 had poor functional outcome, 3 had good to excellent outcome and 3 had satisfactory functional outcome. 4 of these had restricted movements terminally and 2 had pain on movement.

Conclusions: From our study based on patients of clavicle fractures, it can be concluded that the operative group had significantly higher excellent outcomes as compared to the conservatively- managed group, based on the Constant and Murley score.
Keywords: Clavicle Fractures, Constant and Murley Score, Operative Clavicle Fracture Management, Clavicle Fracture Functional Outcome

\section{INTRODUCTION}

Clavicle is a subcutaneous bone, which attaches axial to peripheral skeleton. It is responsible for weight transfer to axial skeletal from shoulder girdle and is instrumental in certain movements of the shoulder joint. Clavicular fractures account for approximately $5-10 \%$ of all fractures. Middle third clavicle fractures are around $70 \%-80 \%$ while lateral third fractures are around $12 \%-15 \%$ and medial third are around $5 \%$ to $8 \%$. [1] It can be caused because of the fall on outstretched hand, or direct trauma.

Treatment modalities of clavicle fracture can be either conservative in the form of brace, figure of 8 bandage, arm sling or operative treatment in the form of Open Reduction and Internal fixation with screws and plate construct using locking, non locking or combination of both.

\section{MATERIAL AND METHODS}

The study was conducted at tertiary level hospital, with the same primary surgeon over a period of 1 year. A total of 40 cases satisfying inclusion and exclusion criteria were included in the study. Alternate patients were allocated to operative and conservation groups. In total 20 patients were operated operatively and 20 patients were treated conservatively.

All 20 patients were evaluated for shoulder and thoracic injuries. Systemic and 
local examinations of the injured extremity were done. On examination the swelling, deformity were checked on inspection and tenderness, abnormal mobility, crepitus were checked on palpation. Skin status evaluation was carried out and examination around the shoulder for associated other injuries/soft tissue injury was done. Then relevant X-rays were taken. Fracture patterns were classified based on the AO/OTA classification. The limb was then immobilised in arm-pouch till definitive mode of management was decided. For surgical group, all the routine laboratory investigations like $\mathrm{CBC}$, RBS, Serum electrolyte, RFT, LFT, PT-INR and ECG, Chest X Rays were done.

\section{Inclusion Criteria}

- All patients visiting Emergency Department or Outpatient Department above the age of 18 years with Fracture of Clavicle

\section{Exclusion Criteria}

- Patients below 18 years of age

- Patients with Delayed union or Non union of the Clavicle

- Patients with Fracture of Medial End of Clavicle

- Patient with co Morbidities Making them unfit for anaesthesia and surgery

- Patient with poor soft tissue conditions

All patients were divided into two groups. Alternate patients irrespective of age, sex and fractures pattern were allocated for conservative and operative treatment. Conservative treatment given was in form of Figure of eight brace or Clavicular brace with arm pouch. The limb was immobilised in such a brace for 6 weeks. Repeated radiographs were taken at 1 week, 4 week and 6 weeks, 12 weeks.

In operative treatment group patients a standard surgical protocol was used. Informed consent of patient was taken. All patients were operated in General Anaesthesia. Patients were put in supine position with a sand bag under injured shoulder. Over the fracture site, a 7-9 centimetres incision was made in the anterior part. Division of the skin, subcutaneous tissue and platysma was done without undermining the edges. The overlying fascia and periosteum were divided next. The osseous ends were freed from surrounding tissue. Minimal soft tissue and periosteal dissection was done. Reduction of the fracture fragments was followed by plate application over the superior clavicular part. The inferior surface was exposed at the junction of the medial and middle third of the clavicle, so that a protective instrument can be inserted during drilling to prevent injury to neuro-vascular structure below it. The plate was fixed to the medial and lateral fragment with a 3.5 millimetres cortical screw and a minimum of three screws were applied in medial and lateral fragments. Wound closure was layered after ensuring proper haemostasis and sterile dressing was applied. ${ }^{[2]}$ Patient was given Intra Venous antibiotics for 3 days after surgery.

Rehabilitation of the affected arm was started on first post- operative day. Gentle pendulum exercises to the shoulder were allowed. At 3 to 4 days gentle active assisted range of motion of the shoulder was allowed but abduction in limited to 80 degrees. At 6 to 8 days active range of motion in all planes were allowed.

Wound inspection was done at 3rd day, sutures removed at 2 weeks. Patients were followed up at 4 weeks, 6 weeks and 12 weeks. Cases were assessed at follow up clinically and radiologically and final results designated as Excellent, Good, Fair and Poor. The functional outcomes were assessed by Constant and Murley score. ${ }^{[3]}$

The functional outcome was assessed by subjective (maximum 35 points) and objective (maximum 65 points) evaluations, with the total scores being allotted out of the maximum 100. Based on the total scores, the functional outcomes were graded as the following: $91-100$ as excellent, 81-89 as good, 71-80 as 
satisfactory, $61-70$ as adequate and $0-60$ as poor.

\section{Statistical Analysis}

Descriptive statistics was applied for analysis by using proportions and percentages. Chi-square test was done to check the association or independence of variables.

\section{RESULTS}

The study was carried out on 40 patients of fresh fracture of the clavicle, out of which 20 were treated conservatively and 20 operatively at our hospital. $36(90 \%)$ of the patients suffered from clavicle fractures due to road traffic accidents while 4 of them fractured their clavicle due to fall on the outstretched hand. Mean age of the patients in the study was 42.4 years. Out of the 40 patients enrolled in the study, $31(72.5 \%)$ were males while $9(27.5 \%)$ were females. All patients were available for follow up and were evaluated clinically in form of tenderness over fracture site and radiographic union. There were associated injuries in few patients with clavicle fracture who suffered road traffic accident. The associated injuries were scapulae fracture $(n=2)$, mandible fracture $(n=1)$, proximal tibia fracture $(n=1)$, Tibia shaft fracture $(n=2)$, Fracture $\operatorname{ribs}(n=5)$. In this study, clavicle fracture on right side was seen in total $30(75 \%)$ patients and $10(25 \%)$ patients had left sided fractures. Based on the Robinson classification of mid-shaft clavicle fractures, majority of patients were in B1 (18 i.e. 45\%) group followed by A2 (09 i.e. $22.5 \%)$, B2 (08 i.e.20\%), A1 (5 i.e. $12.5 \%)$. Functional outcome of each patient was noted at 1 month. From the total of 40 patients, $28(70 \%)$ had excellent or good functional outcome at 4 weeks irrespective of the treatment. 7 of the 20 patients who were managed conservatively had satisfactory to poor scores as compared to 3 patients out of 20 who were managed operatively. The functional outcome results were assessed by the Constant and Murley Score. The number of patients in the operative group who showed excellent results were significantly more than that in the conservative group $(\mathrm{p}=0.0324)$.

A total of 23 patients in the study suffered from un-displaced fractures, 10 of which were managed conservatively while 13 were managed operatively. In this group with un-displaced clavicle fracture, 11 patients showed good to excellent outcome based on Constant and Murley Score, of which 7 were treated conservatively (35\% of conservatively managed patients) and 4 were operatively managed $(20 \%$ of the operated patients). Out of the 20 operated patients, all patients were operated using pre-contoured locking plate.

In this study, 20 the patients who were operated upon had an average union time of 8.4 weeks; 1 patient had delayed fracture union by 12 wks. 16 patients in conservative group had normal union of fractures with 3 patients had delayed union and 1 patient going into non-union. Average union time in the conservative group was 10.6 weeks, which was more than the 8.4 weeks seen in the group treated operatively. Mal-union was present in 8 of the 20 patients treated conservatively, and 6 of these 8 had a visible deformity. Out of these patients with mal-union 1 had poor functional outcome, 3 had good to excellent outcome and 4 had satisfactory functional outcome. 4 of these had restricted movements terminally and 2 had pain on movement. In surgically treated group, 1 patient suffered from superficial infection while another 1 experienced implant failure in forms of loss of reduction and implant separation from distal fragment. Skin related complication included keloid formation over scar for open reduction and plating was seen in one patient.

\section{DISCUSSION}

A total of 40 cases with clavicle fractures were selected for the study. Of these 20 patients were managed by nonoperative methods and another 20 patients by operative methods. In this study, the average age of the patient was 32.90 
years, with the youngest patient being 17 years and the oldest being 57 years old, among them, male predominance was present $(83.33 \%)$ and female were $16.66 \%$. Pearson et al. ${ }^{[4]}$ have reported the average age of patients sustaining a clavicular fracture is 33 years. Postacchini et al. ${ }^{\text {[5] }}$ reported that most patients were men (68\%).

In this study, $63.33 \%$ developed clavicle fracture following RTA with direct impact on the shoulder girdle, followed by fall on out stretched hand in $30 \%$, physical assault in $3.33 \%$ and blunt trauma in $3.33 \%$. Zlowodzki et al. ${ }^{[6]}$ and McKee et al. ${ }^{[7]}$ described a fall or a blow to the shoulder, giving an axial compressive force on the clavicle, is the most common trauma mechanism of injury for any clavicular fracture.

In our study, $56.66 \%$ had clavicle fractures on the left side. Postacchini et al. [5] also described that the left side was involved in $61 \%$ of cases. Associated injuries commonly noted in this study were rib fractures (13.33\%), abrasions (13.33\%), fracture both bone leg (6.66\%), scaphoid fracture $(3.33 \%)$, glenoid neck fracture $(3.33 \%)$ and tibial plateau fracture $(3.33 \%)$. Associated injuries have been reported in different studies. ${ }^{[2-8]}$

In our series, $86.3 \%$ had mid-third fracture, $13.7 \%$ had lateral third fracture. Mid-third fracture of the clavicle has been found to be more common in different studies. ${ }^{[5-9]}$

Patients in the operative group improved functionally and returned to normal activities earlier than nonoperative group. It is also statistically significant $(\mathrm{P}<$ $0.05)$. This factor is very important as patients today are more active and expect to return to pain-free function following a fracture.

Patient satisfaction in the operative group is more than in the nonoperative group regarding appearance of the shoulder (cosmetic) and movements of the shoulder $(\mathrm{P}=0.039)$. With conservative treatment for displaced and shortened mid-shaft clavicle fractures, Thyagarajan et al. ${ }^{[8]}$ reported less satisfaction among patients. The mean time for fracture healing (radiological union) was shorter in the operative group (8.4 weeks) than nonoperative group (10.6 weeks). McKee et al. ${ }^{[7]}$ described the mean time for fracture healing were 14-16 weeks for operated patients and 24-28 weeks for non operated patients.

The complications were more in the nonoperative group like symptomatic malunion 8 cases (40\%), shortening 3 cases $(15 \%)$, muscle wasting 4 cases $(20 \%)$, pressure necrosis 1 case (5\%) and complex regional pain syndrome 1 cases $(5 \%)$. The complications noted in the operative group were incisional numbness 1 case $(5 \%)$ and hardware irritation 3 case $(15 \%)$. Second surgery was done to remove irritating hardware. None of the operated patients had nonunion or malunion.

Iatrogenic neurovascular vascular injury is an imminent complication if proper operative techniques are not followed. Because major neurovascular structures like subclavian vein, subclavian artery and brachial plexus are near to the surgical field. [9] However, in this study, none of our operated patients developed any neurovascular injury. None of the patients in this study had pulmonary injury either following primary injury or iatrogenically.

Functional outcome was assessed using the objective and subjective parameters of the Constant and Murley score. In our study, the functional outcome was excellent in 15 cases $(37.5 \%)$, good in 12 patients $(30.0 \%)$, satisfactory in 4 cases $(10 \%)$, adequate in 1 participant $(2.5 \%)$ and poor in 8 patients $(20 \%)$. Out of all the excellent outcomes, 5 cases were from conservative group while 10 patients in operative group. On Fisher's exact test, it was found that the difference between the number of excellent outcomes between the two groups was significant $(p<0.05)$. A previous study states that though the nonoperative group did approach the functional outcome of the operative group at 18 months, a significant difference was still evident. ${ }^{[8]}$ 
In our study, the 20 patients who were operated upon showed an average union time of 8.4 weeks. Only 1 patient had union delayed by 12 weeks. In the conservatively managed group, 18 patients reported normal fracture union, 1 patient had delayed union and another 1 patient had non-union. Average union time in conservative group was 10.6 weeks. It was noteworthy that malunion was a major concern in the conservatively managed group, with 8 out of 20 patients (40\%) suffering from it. In a previous study, all fractures in the operative group showed normal union, whereas eight fracture patients in the non-operative group reported non-union $(29 \%)(\mathrm{P}=0.002)$. In addition, 10 patients $(35.7 \%)$ in the non-operative group experienced a symptomatic malunion compare with two (4.4\%) surgically treated patients. ${ }^{[8]}$

\section{CONCLUSION}

From our study based on patients of clavicle fractures, it can be concluded that the operative group had significantly higher excellent outcomes as compared to the conservatively- managed group, based on the Constant and Murley score. The number of mal-union reported in the conservatively treated group was significantly more with that in the operative group. The average union time observed in the non-operative group was more as compared to that in the operative group. Thus, clavicle fractures managed with operative modalities had a better outcome with much lesser postoperative complications.

\section{REFERENCES}

1. Craig EV, Basamania CJ, Rockwood CA. Fractures of the clavicle. Chapter-11, In : Rockwood CA, Matsen FA, Wirth MA, Lippitt SB, editors, The shoulder. 3rd edition Philadelphia: Saunders 2004, 455519.

2. Lazarus MD. Fractures of the Clavicle. Chapter-26, In: Bucholz RW and Heckman JD, editors, Rockwood and Green's fractures in adults, 5th edition, Philadelphia: Lippincott Williams and Wilkins. 2001; 1041-78.

3. Constant CR, Murley AHG. A clinical method of functional assessment of the shoulder. Clinical Orthopaedics and Related Research. 1987;214:160-4.

4. Pearson AM, Tosteson AN, Koval KJ, McKee MD, Cantu RV, Bell JE, et al. Is surgery for displaced, midshaft clavicle fractures in adults cost-effective. Results based on a multicenter randomized, controlled trial. J Orthop Trauma 2010;24: 426-33.

5. Postacchini F, Gumina S, De Santis P, Albo F. Epidemiology of clavicle fractures. J Shoulder Elbow Surg 2002;11:452-6.

6. Zlowodzki M, Zelle BA, Cole PA, Jeray K, McKee MD. Treatment of acute midshaft clavicle fractures: Systematic review of 2144 fractures. J Orthop Trauma 2005;19: 504-7.

7. McKee MD, Pedersen EM et al. Deficits following nonoperative treatment of displaced midshaft clavicular fractures. $\mathbf{J}$ Bone Joint Surg Am 88(1): 35-40.

8. Gille J, Schulz A, Wallstabe S, Unger A, Voigt C, Faschingbauer M. Hook plate for medial clavicle fracture. Indian $\mathbf{J}$ Orthop 2010;44:221-3.

9. Thyagarajan DS, Day M, Dent C, Williams R, Evans R. Treatment of mid-shaft clavicle fractures: A comparative study. Int J Shoulder Surg 2009;3:23-7.

10. Kulshrestha V, Roy T, Audige L. Operative versus nonoperative management of displaced midshaft clavicle fractures:

How to cite this article: Phanswal V. Comparative study of conservative vs surgical management of clavicle fractures. Gal Int $J$ Health Sci Res. 2021; 6(1): 1-5. 